and practice development. Nurses and family carers are faced with different challenges when providing care to patients at the end of life. Therefore, it became imperative to explore the factors perceived to promote care at the end of life from the perspective of nurses, patients, and family carers.

Method A qualitative ethnographic case study design was employed for the study. Participant observations consisting of 188 hours of observation, and semi-structured interviews with 40 participants (nurses-14, patients-12, and family carers-14) were used as tools for data collection. Data analysis follows an inductive thematic approach using NVivo 11 qualitative data management programme.

Findings Six overarching themes emerged: Enhanced communication skills, Increase drug supply, improve manpower, reward or incentives, training of staff, and improve logistics supplies.

Conclusion Basically, these factors were directly related to the poor managerial processes and hospital's policies. If the hospital management increases drug supply, improve manpower, revive decayed infrastructure, improve logistics supplies, provide reward or incentives to staff, and encourages staff training and development, certainly the quality of palliative and end of life care will be improved.

\section{AN ANALYSIS OF THE QUALITY OF ADVANCED CARE PLAN AND DNACPR DOCUMENTATION PRIOR TO THE INTRODUCTION OF THE RESPECT PROCESS}

Kiran Parmar, Emma Wilkinson, Sinead Henderson, Clare Smith. St Peter's Hospital, Ashford and St Peter's Hospitals NHS Foundation Trust, Chertsey, Surrey

10.1136/bmjspcare-2019-ASP.81

An Advanced Care Plan (ACP) allows discussion and documentation of patient preferences for their future medical care which is not limited to resuscitation only. Despite national guidance, there remains poor communication and inconsistent documentation of ACP and DNACPR decisions, potentially resulting in inappropriate treatment and hospital admissions for patients. ReSPECT (Recommended Summary Plan for Emergency Care and Treatment) is a newly introduced emergency care plan which aims to help support advanced care discussions and record recommendations. We aimed to assess the quality of ACP and DNACPR documentation at a UK District General Hospital before introducing the ReSPECT form.

Retrospective data was collected on all patients from two elderly care wards in March 2018 through case note review. Section headings on the ReSPECT form were used as the basis for collection. Data was also collected on ACP/DNAR decision communication on discharge documentation.

87 patients (52 male and 35 female). 25\% (22) had ACP and $72 \%$ (63) had DNACPR agreed. Those with DNACPR, $79 \%$ (50) had discussion documented in clinical notes, and $57 \%$ (36) on discharge documentation. Those with ACP agreed, 68\% (15) of discussions were documented in clinical notes, 95\% (21) had ACP on discharge documentation completed by junior doctors.

ACP and DNACPR decisions are individual to each patient and our initial data shows they are not always clearly documented. Interestingly, ACP decisions are found more on discharge summaries than recorded in medical notes. Discharge summaries are completed by junior doctors and therefore need support by clear senior decision making in the notes. This is currently missing for $27 \%$ of ACP decisions and highlights that discussions required for the ReSPECT process are not currently routinely documented. The ReSPECT form alongside web-based training was introduced in October 2018. The next step is to re-audit and assess its impact on documentation and communication.

\section{PLANNING AHEAD: RETURNING HOME ON VENTILATORY SUPPORT FOR END-OF-LIFE CARE}

Jessica Walding, Meg Williams, Joanne Evans, Edward Curtis, Alison Stevens. Aneurin Bevan NHS Trust

\subsection{6/bmjspcare-2019-ASP.82}

Background The Intensive Care National Audit and Research Centre UK (ICNARC) revealed that approximately 20\% of patients admitted to the Intensive Care Unit (ICU) will die there. End of life care decisions are a fundamental aspect of care on ICU, particularly given that $70 \%$ of the deaths that occur are following the withholding or withdrawal of life-sustaining treatments. Palliative care is increasingly accepted as an essential component of comprehensive care for critically ill patients. Most deaths are managed independently on the ICU, as such, patients preferred place of death (PPD) is not often explored or fully established.

Aims

1. To highlight the importance of end-of-life care and early palliative care team input to everyday practice and training for anaesthetists and intensivists.

2. To actively consider whether transfer home may be a component of end-of-life care for some patients.

Methods Our patient population are those inpatients receiving additional respiratory support on the ICU, non-invasive or invasive ventilation, who's expressed their PPD as home. We recognise that collaborative and innovative working between teams within the acute and community sector is key to facilitating a safe and efficient transfer home for end-of-life care for patients requiring ventilatory support.

Results At our hospital, we have facilitated two patient transfers from ICU to home for withdrawal of ventilatory support and end-of-life care. Following these transfers, a comprehensive discharge checklist has been implemented, with key focus to ensuring a safe but time efficient transfer.

Conclusions To conclude, we are not asserting that transfer home at end-of-life is desirable or feasible for all critically unwell patients. However, we want to highlight the important of exploring patient wishes at this crucial stage of patient care.

\section{A REVIEW OF CODE STATUS DOCUMENTATION IN PATIENTS REFERRED TO THE PALLIATIVE CARE INPATIENT CONSULT SERVICE}

Sheena Geoghegan, Fiza Warsi, Sara Kleinschmidt, Reid Hartmann, Douglas Smucker. The Christ Hospital, Cincinnati, Ohio

\subsection{6/bmjspcare-2019-ASP.83}

Background Inpatient Palliative Care consult services see a wide range of acutely admitted patients, many of whom are critically ill. Due to the nature of an acute admission, code status clarification or discussion is often overlooked and can affect patient's clinical course and outcome. 
Objectives

1. To investigate the documentation of code status discussion on admission to hospital.

2. To investigate patient outcomes in those referred to Palliative care services.

Methods The electronic health records (EHR) of 50 patients referred to the Palliative Care consult service were retrospectively reviewed, following Institutional Review Board (IRB) approval. The patients code status orders, clinical notes, and outcomes were documented.

Results The average age of the patients was 67.86 years. $48 \%$ had an active cancer diagnosis, $10 \%$ were left ventricular device (LVAD) patients and $42 \%$ had an acute cardiac, pulmonary or neurological presentation. $34 \%$ of patients were admitted directly to an intensive care setting. The majority $(80 \%)$ of patients were 'Full Code' on admission, 12\% were 'Do Not Resuscitate' (DNR) and 8\% were 'Partial Code'. For those who were documented as 'DNR', only 1 patient had a documented 'Do Not Intubate' (DNI) order. While code status was documented in all admission notes, the discussion of code status clarification was only documented in $14 \%$ of notes. Only one emergency department note referenced a discussion of code status. Code status was changed within 24 hours in $6 \%$ of patients, and $20 \%$ of patients changed code status during admission, with Palliative Care involvement in the majority $(80 \%)$ of these cases. Regarding outcomes, 56\% returned home, 14\% were discharged for rehabilitation, $22 \%$ were discharged with hospice care, and $6 \%$ died in hospital on comfort care.

Conclusion Code status discussion should be an integral part of an acute hospital admission to ensure patient's wishes are being followed and respected.

\section{COMPLIANCE WITH HOSPITAL GUIDELINES ON PRESCRIPTION OF ANTICIPATORY MEDICATIONS - SCOPE FOR IMPROVEMENT?}

Manisha Patel, Sally Sutcliffe, Mariam George. Tameside and Glossop Integrated Care Foundation Trust

\subsection{6/bmjspcare-2019-ASP.84}

Background NICE guidelines for the Care of the Dying Adult recommend the prescription of anticipatory medication as early as possible to minimise symptomatic distress during last days of life. Literature on prescribing of anticipatory medications in secondary care is limited. Incorrect prescribing and inadequate understanding among hospital staff regarding symptom management for a dying patient prompted the need to establish compliance of prescribing and administration of anticipatory medications (AMs) against Trust guidelines on symptom control in the last days of life.

Method A retrospective audit cycle comprising of 3 audits was conducted examining hospital records of patients recognized as in the dying phase, between December 2015 and October 2017. Documentation within drug charts, doctors' and nursing notes were scrutinised to establish whether AMs were prescribed and used for specific indications as per guidelines.

Results There was improvement in compliance with prescribing of AMs for patients at point of recognition of being in the dying phase $(88 \%$ to $96 \%)$ and the administration of AMs according to correct indications (23\% to 91\%). Nursing documentation of indication for AM use improved (23\% to 40\%). Despite an improvement in selection of appropriate AM per symptom, compliance around doctors' prescription of accurate doses dropped (21\% to $12 \%)$.

Conclusions Whilst compliance in the initiation of anticipatory medications for the main symptoms associated with last days of life improved, accuracy of doses needs to improve. The role of hospital pharmacists in enabling compliance was identified as an additional resource alongside periodic trust-wide communication of guidelines, to support better symptom control at end of life. The prevalence of hospital staff turnover (doctors changing rotations and bank nurses) necessitates the need for regular educational events to facilitate safe, evidence based symptom control at end of life. Embedding clinical guidelines for end of life care requires an ongoing proactive approach.

\section{2 'PLEASE, I WANT TO GO HOME' - CREATING A HOMECARE TEAM TO EXPEDITE DISCHARGE HOME FROM HOSPITAL AT THE END OF LIFE}

Magdalen Thomson, Sarah McGaughey, Tracey Platt, Rachel Gaffney. Hampshire Hospitals NHS Foundation Trust

\subsection{6/bmjspcare-2019-ASP.85}

Background The project addressed delays in discharge and its impact on unnecessary acute bed occupancy, responding to 'Please, I want to go home' for dying patients. The press perceptions that the 'NHS must get a grip on hospital discharge delays', and discharge delay rise by $23 \%$ in the preceding year (NHS England) led to agreement that it was unacceptable that vulnerable and dying patients were 'stuck' in hospital.

Method A case for change put to our local CHC commissioners highlighted the lack of care capacity, CCG spot purchasing methodology, and patients with agreed fast track (FT) funding being inappropriately admitted into hospital as no care available at home. FT applications were increasing yearly, the CHC department was understaffed and challenged to meet the NHS CHC Framework timeframes, and the FT reviews were not being undertaken within 3 months. Some individuals had been on the FT pathway for 3 years. Commissioner contract aims were agreed assuring quality of service provision, a responsive service to meet individual needs, implementation within 48 hours, and over seen by the hospital palliative care team. Continuity of care, review of FT eligibility and dynamic care delivery, assured best value of NHS CHC resources whilst improved patient experience.

Results In March 2016 The Homecare service was set up within Winchester and Andover, funded by $\mathrm{CHC}$ fast track. The first patient was accepted on 18th July 2016.

In the first 6 months the service supported 32 patients, delivered 1,147 hours of care by Band 3 healthcare support workers 8 am to $8.30 \mathrm{pm}$, and supported by the hospital/hospice palliative care team. Fast track discharge delays were reduced from 15.3 days to less than 2 days.

Conclusion This service enabled swift patient discharge, met preference, and provided timely, high quality, flexible care. It also improved patient flow through Andover and Winchester hospitals. 